\title{
Kinematical data on early-type galaxies. VI. ${ }^{\star \star \star}$
}

\author{
F. Simien and Ph. Prugniel \\ CRAL-Observatoire de Lyon, CNRS: UMR 142, 69561 St-Genis-Laval Cedex, France \\ Received 8 June 2001 / Accepted 7 December 2001

\begin{abstract}
We present the result of spectroscopic observations of a sample of 73 galaxies, completing the database published in this series of articles. The sample contains mostly low-luminosity early-type objects, including four dwarfs of the Local Group (in particular, deep spectra of NGC 205), 15 dEs or dS0s in the Virgo cluster, and UGC 05442, a spheroidal dwarf of the M 81 group. We have measured the central velocity dispersion for all but one object, and determined the major-axis rotation and velocity-dispersion profiles for 59 objects. For the current sample of diffuse (or dwarf) elliptical galaxies, we have compared stellar rotation to velocity dispersion; the analysis suggests that these objects may be nearly rotationally flattened, and therefore that anisotropy may be less important than previously thought.
\end{abstract}

Key words. galaxies: elliptical and lenticular, $\mathrm{cD}$ - galaxies: kinematics and dynamics - galaxies: fundamental parameters - galaxies: general

\section{Introduction}

We have presented kinematical measurements from absorption spectroscopy on early-type galaxies (Simien \& Prugniel 1997a, 1997b, 1997c, 1998, 2000: hereafter Papers I to V, respectively); these data are intended to contribute to the study of several structural and evolutionary issues. This material allowed us to assess the contribution of rotation to the energy balance in earlytype galaxies through the analysis of the residuals to the Fundamental Plane (FP, Prugniel \& Simien 1994). In addition, the accurate kinematical measurements gathered have permitted us to further develop the interpretation of the residuals to the FP. We concluded (Prugniel \& Simien $1996,1997)$, that the tilt of the FP is fully accounted for by the combined effects of: a) the differences in stellar population, $\sim 50 \%$ in the $B$ band, b) the rotational support, $\sim 25 \%$ with an enhanced contribution in small galaxies and, c) the non-homology of the spatial structure, $\sim 25 \%$. Age effects, i.e., the presence of an intermediateage sub-population in about $10 \%$ of our galaxies (in the field, in groups and poor clusters), result in a skewness of

Send offprint requests to: F. Simien, e-mail: simien@obs . univ-lyon1.fr

* Based on observations collected at the Observatoire de Haute-Provence.

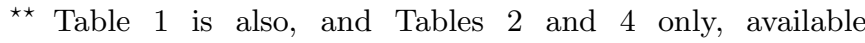
in electronic form at the CDS via anonymous $\mathrm{ftp}$ to cdsarc.u-strasbg.fr $(130.79 .128 .5)$ or via http://cdsweb.u-strasbg.fr/cgi-bin/qcat?J/A+A/384/371 the residuals, and they are correlated with the density of the environment (Prugniel et al. 1999).

In the general framework of galaxy formation and evolution, the transition between the properties of elliptical galaxies and those of diffuse, or dwarf, ellipticals (dEs), is pivotal. Indeed, these two classes populate different regions of the FP, and this has been interpreted as evidence of a fundamental difference in their formation and evolution processes (see, e.g., Bender et al. 1992 and references therein: hereafter BBF92); an observed difference in the kinematical status has been claimed, with intermediate ellipticals being isotropic and dwarfs anisotropic, and this has been considered as supporting the above evidence (BBF92). The actual nature of the kinematical transition between Es and dEs, therefore, has major implications.

So far, this transition has not been addressed in a comprehensive way. Long-slit kinematics along the major axis were available for little more than a dozen dEs (Bender et al. 1991, hereafter BNP91; Bender \& Nieto 1990; Peterson \& Caldwell 1993); comparisons of rotation to dispersion suggested significant anisotropy, but these measurements were generally close to the limit of the instrumental possibilities. We wanted to revisit this question, starting with better-adapted observations; we have taken advantage of the low read-out noise of recent CCDs to increase the spectral resolution, and to extend the profiles to fainter surface brightness for a more global view of the kinematics. In this sixth paper of the series, we continue the presentation of all our spectroscopic data but, for the first time, the recent runs include a high fraction 
of dwarfs galaxies. For this class of objects, the present set, although still very limited, has been found sufficient for a preliminary analysis of the kinematical behavior.

The paper is organized as follows. Section 2 describes the observations and the sample characteristics. Section 3 is devoted to the data reduction, the presentation of the results, and their evaluation. Section 4 discusses the results, the characteristics of several individual objects, and the kinematical test on the dwarfs.

\section{Observations and sample characteristics}

The observations were secured at the $1.93-\mathrm{m}$ telescope of the Observatoire de Haute-Provence, equipped with the CARELEC long-slit spectrograph. For the first run presented here (March 1998), the receptor was still the $512 \times 512$ Tektronix CCD used for the precedent papers of the series; the selected setup (hereafter setup \#1) provided a spectral range of $\simeq 900 \AA$ centered on $\lambda=5200 \AA$ with a $F W H M$ resolution of $\simeq 3.2 \AA$ (reciprocal resolution: $R=1625$ ) corresponding to an instrumental velocity dispersion of $\simeq 82 \mathrm{kms}^{-1}$. The slit width, projected onto the plane of the sky, was typically $2.2^{\prime \prime}$. For the subsequent runs, we have used a new EEV receptor with $2048 \times 780$ pixels of $15 \mu \mathrm{m}$, and we selected a grating providing an angular dispersion twice as large; with this setup (\#2), the available wavelength range was the same, and the instrumental velocity dispersion was $\simeq 25 \mathrm{~km} \mathrm{~s}^{-1}$ $(R=5050)$ for a slit width of $1.5^{\prime \prime}$.

With the setup adapted to low-dispersion galaxies (\#2), the limiting surface brightness, mostly dependent on the night-sky level, is typically in the range from $\mu_{V}=22$ to $23 \mathrm{mag} \operatorname{arcsec}^{-2}$, for a set of four combined 50-min exposures.

From March 1998 to January 2001, seven observing runs totalled 35 clear (or partially clear) nights. The atmospheric conditions were variable, with a seeing disk between $2^{\prime \prime}$ and $3.5^{\prime \prime}$ (FWHM) for most objects, but up to $6^{\prime \prime}$ for two of them. These variations are an additional justification for the repeat measurements on several objects. The log of the observations is given in Table 2, which is proposed in electronic form only.

For the first run, we mostly continued our program targeted on intermediate-luminosity early-type galaxies. For the subsequent runs, taking advantage of the new instrumental setup, a high fraction of the observing time was devoted to dwarfs. For most objects, at least two spectra were collected along the major axis or an axis close to it, with an individual exposure time between 45 and $60 \mathrm{~min}$. The dwarfs included 15 early-type objects in the Virgo cluster, classified dE or dS0 by Binggeli \& Cameron (1991: hereafter BC91), with $-17.6 \leq M_{B} \leq-15.6$. For most dwarfs, three or four spectra were secured.

As interesting, nearby examples of dwarfs, we observed NGC 147, NGC 185, and NGC 205, together with NGC $221 \equiv$ M 32 as the archetype of compact objects. For three of these objects, the cumulated exposure time was standard, but was much longer for NGC 205. In this special case, our aim was to get a deep rotation curve, reaching regions fainter than $\mu_{V}=22 \mathrm{mag} \operatorname{arcsec}^{-2}$, where the flux and noise were dominated by the nightsky contribution; in order to bypass the limitations of the $5^{\prime}$ slit length, we off-centered the nucleus and secured five 50-min spectra on each side; forecasting the interest of a complete set of kinematical data, we also made six exposures on the minor axis.

The most extreme case is UGC 5442, a faint dwarf whose central surface brightness is $\mu_{V}=$ $23.3 \mathrm{mag} \operatorname{arcsec}^{-2}$ (Karachentsev et al. 2000), for which we accumulated 14 one-hour exposures, in an attempt to resolve the galaxy from the noise.

Our present sample gathers 73 objects, most of them early-type galaxies, with also eight spirals which we had observed within the frame of another project (kinematics and stellar populations in bulges, see Prugniel et al. 2001). These bulge spectra are aimed at providing several line indices in addition to the kinematical parameters, for the continuation of the above-mentioned project; we have included them in the present paper for the sake of homogeneity: as in the preceding papers of the series, each observing run was reduced globally, in order to ensure consistency.

Relevant catalog elements are presented in the first nine columns of Table 1 . The ellipticals span almost the entire flattening range, and the lenticulars are moderately to highly flattened. Most distances are within $\simeq 25 \mathrm{Mpc}$ (for $H_{0}=75 \mathrm{~km} \mathrm{~s}^{-1} \mathrm{Mpc}^{-1}$ ), with only a couple of objects farther away. The absolute magnitudes are in the range $-21.1 \leq M_{B} \leq-13.5$.

\section{Data reduction and result evaluation}

\subsection{Technique summary}

As in Papers I to V, standard pre-processing was applied to the raw data, up to the rebinning in wavelength. The galaxy centers $(r=0)$ were determined by a Gaussian fitting to a limited range $\left(\simeq 12^{\prime \prime}\right)$ around the intensity peak. In the outer regions, cosmic-ray hits were removed with a median filter, and adjacent lines were combined with a variable weighting function (a Gaussian continuously wider faint-ward). A Fourier-Fitting technique determined the central velocity dispersion $\sigma_{0}$ and, when possible, the radial profile $\sigma(r)$ of the dispersion, together with the projected rotation curve $V(r)$ along the major axis. A twopass mode (described in Paper I) allowed us to remove cosmic-ray hits on the inner lines, where the spatial resolution must be preserved. We adopted as $\sigma_{0}$ the dispersion measured on the line at $r=0$, or an interpolation between the values on the two lines bracketing the position $r=0$; similarly, the systemic velocity corresponds to $r=0$. Whenever possible, we have determined the maximum rotation velocity $V_{\max }$, as the mean of representative values along opposite semi-axes. 
Table 1. Catalog elements and kinematical results.

\begin{tabular}{|c|c|c|c|c|c|c|c|c|c|c|c|c|}
\hline Object & Type & $\alpha_{1950}$ & $\delta_{1950}$ & $B_{\mathrm{T}}$ & $-M_{B}$ & $r_{\mathrm{e}}$ & $\epsilon$ & $\mathrm{PA}$ & $c z$ & $\sigma_{0}$ & $V_{\max }$ & $r_{\max }$ \\
\hline$(1)$ & $(2)$ & $(3)$ & $(4)$ & $(5)$ & (6) & $(7)$ & $(8)$ & $(9)$ & $(10)$ & $(11)$ & $(12)$ & $(13)$ \\
\hline IC 0225 & $\bar{E}$ & 022353.9 & +005611 & 14.83 & 16.61 & $\ldots$ & 0.12 & 175 & $1543 \pm 11$ & $22 \pm 06$ & $<11 \pm \ldots$ & 10 \\
\hline IC 0781 & $\mathrm{dS} 0(4), \mathrm{N}$ & 121730.6 & -151417 & 14.14 & 17.35 & $\ldots$ & 0.10 & 30 & $1364 \pm 09$ & $35 \pm 04$ & $14 \pm 05$ & 10 \\
\hline IC 0783 & $\mathrm{SAB}(\mathrm{rs}) 0 / \mathrm{a} ?$ & 121906.8 & +160123 & 14.64 & 16.65 & 17.4 & 0.12 & 110 & $1267 \pm 12$ & $36 \pm 10$ & $22 \pm 09$ & 15 \\
\hline IC 0783A & $\mathrm{SB}(\mathrm{r}) 0 ?$ & 121947.9 & +160036 & 15.40 & 17.41 & $\ldots$ & 0.17 & 4 & $1207 \pm 12$ & $30 \pm 08$ & $\ldots \pm \ldots$ & $\ldots$ \\
\hline IC 1211 & $\mathrm{E}$ & 161538.6 & +530740 & 13.40 & 21.05 & 18.2 & 0.07 & 34 & $5618 \pm 19$ & $198 \pm 17$ & $102 \pm 16$ & 20 \\
\hline IC 3120 & S0? & 121542.6 & +140136 & 15.74 & $\ldots$ & $\ldots$ & 0.09 & 0 & $254 \pm 12$ & $26 \pm 16$ & $\ldots \pm \ldots$ & $\ldots$ \\
\hline IC 3328 & $\mathrm{dE}, \mathrm{N}$ & 122324.6 & +101947 & 14.01 & 17.28 & 21.9 & 0.08 & 83 & $1025 \pm 10$ & $38 \pm 03$ & $16 \pm 05$ & 25 \\
\hline IC 3381 & $\mathrm{dE}, \mathrm{N}$ & 122543.0 & +120358 & 14.31 & 15.58 & $\ldots$ & 0.24 & 99 & $675 \pm 12$ & $45 \pm 07$ & $<18 \pm \ldots$ & 12 \\
\hline IC 3393 & $\mathrm{dE}, \mathrm{N}$ & 122609.8 & +131131 & 15.02 & 16.27 & 15.8 & 0.59 & 133 & $476 \pm 10$ & $29 \pm 04$ & $10 \pm 07$ & 18 \\
\hline IC 3413 & $\mathrm{dS} 0, \mathrm{~N}$ & 122651.0 & +114230 & 14.15 & 17.06 & $\ldots$. & 0.39 & 169 & $1335 \pm 12$ & $69 \pm 07$ & $13 \pm 08$ & 17 \\
\hline IC 3457 & E3: & 122919.5 & +125559 & 14.19 & 17.10 & 25.7 & 0.29 & 133 & $1297 \pm 12$ & $18 \pm 12$ & $12 \pm 07$ & 18 \\
\hline IC 3461 & $\mathrm{dE}, \mathrm{N}$ & 122930.6 & +120953 & 15.14 & 15.78 & $\ldots$ & 0.17 & 150 & $1001 \pm 11$ & $44 \pm 05$ & $13 \pm 09$ & 12 \\
\hline IC 3468 & $\mathrm{E} 1, \mathrm{~N}$ & 122942.0 & +103129 & 13.68 & 17.62 & 24.5 & 0.10 & 160 & $1288 \pm 10$ & $36 \pm 04$ & $<9 \pm \ldots$ & 18 \\
\hline IC 3653 & E3 & 123844.4 & +113942 & 14.32 & 16.97 & 6.8 & 0.23 & 0 & $567 \pm 10$ & $51 \pm 03$ & $\ldots \pm \ldots$ & $\ldots$ \\
\hline IC 3773 & $\mathrm{~d}: \mathrm{SO}(9)$ & 124444.0 & +102836 & 13.80 & 17.49 & 17.4 & 0.61 & 21 & $1084 \pm 12$ & $40 \pm 03$ & $23 \pm 09$ & 20 \\
\hline Mrk 996 & $\ldots$ & 012504.5 & -063508 & 14.95 & 16.62 & $\ldots$ & 0.27 & 23 & $1602 \pm 11$ & $23 \pm 10$ & $<15 \pm \ldots$ & 10 \\
\hline NGC 0147 & E5 pec & 003027.4 & +481355 & 9.86 & 14.02 & 190.5 & 0.39 & 25 & $-83 \pm 14$ & $45 \pm 17$ & $\ldots \pm \ldots$ & $\ldots$ \\
\hline NGC 0185 & E3 pec & 003612.0 & +480350 & 9.54 & 14.28 & 89.1 & 0.14 & 35 & $-208 \pm 10$ & $20 \pm 04$ & $\ldots \pm \ldots$ & $\ldots$ \\
\hline NGC 0205 & E5 pec & 003738.7 & +412443 & 8.61 & 15.44 & 147.9 & 0.39 & 0 & $-256 \pm 08$ & $20 \pm 01$ & $13 \pm 02$ & 50 \\
\hline NGC 0221 & cE2 & 003957.7 & +403529 & 8.49 & 15.66 & 32.4 & 0.27 & 170 & $-203 \pm 10$ & $79 \pm 01$ & $36 \pm 01$ & 10 \\
\hline NGC 1138 & SB0 & 025319.0 & +425048 & 13.18 & 19.40 & $\ldots$ & 0.11 & 90 & $2335 \pm 11$ & $145 \pm 04$ & $<25 \pm \ldots$ & 20 \\
\hline NGC 1465 & $\mathrm{~S} 0 / \mathrm{a}$ & 035022.2 & +322043 & 13.51 & 20.26 & $\ldots$. & 0.72 & 165 & \pm 38 & $306 \pm 35$ & $\ldots \pm \ldots$ & $\ldots$ \\
\hline NGC 2314 & E3 & 070353.7 & +752428 & 12.91 & 20.78 & 12.0 & 0.18 & 91 & $3840 \pm 14$ & $272 \pm 09$ & $40 \pm 19$ & 20 \\
\hline NGC 2679 & SB0: & 084829.8 & +310312 & 13.39 & 18.90 & $\ldots$ & 0.00 & 172 & $2050 \pm 11$ & $84 \pm 04$ & $\ldots \pm \ldots$ & $\ldots$ \\
\hline NGC 3073 & SAB0- & 095728.8 & +555138 & 13.98 & 17.92 & 10.2 & 0.10 & 90 & $5 \pm 10$ & $36 \pm 04$ & $<10 \pm 06$ & 13 \\
\hline NGC 3226 & E2: pec & 102043.5 & +200906 & 12.01 & 19.99 & 41.7 & 0.19 & 15 & $1313 \pm 16$ & $201 \pm 08$ & $50 \pm 13$ & 30 \\
\hline NGC 3377 & E5-6 & 104502.6 & +141451 & 10.88 & 20.50 & 41.7 & 0.39 & 41 & $4 \pm 14$ & $156 \pm 03$ & $97 \pm 08$ & 38 \\
\hline$N G C 3870$ & S0? & 114316.9 & +502842 & 13.25 & 18.04 & 9.1 & 0.19 & 15 & $743 \pm 10$ & $29 \pm 06$ & $14 \pm 04$ & 15 \\
\hline$N G C 4008$ & E5 & 115542.9 & +282815 & 12.81 & 20.74 & 18.6 & 0.45 & 162 & $3637 \pm 32$ & $240 \pm 35$ & $>123 \pm \ldots$ & 10 \\
\hline$N G C 4121$ & $\mathrm{E}$ & 120526.2 & +652341 & 14.25 & 18.76 & 7.4 & 0.12 & 101 & $4 \pm 12$ & $81 \pm 03$ & $66 \pm 07$ & 09 \\
\hline$N G C 4143$ & $\mathrm{SAB}(\mathrm{s}) 0$ & 120705.0 & +424851 & 11.92 & 19.27 & 17.4 & 0.35 & 144 & $58 \pm 16$ & $211 \pm 06$ & $199 \pm 04$ & 49 \\
\hline NGC 4203 & SABO-: & 121234.1 & +332832 & 11.25 & 19.01 & 39.8 & 0.29 & 30 & $1069 \pm 11$ & $162 \pm 05$ & $69 \pm 06$ & 42 \\
\hline NGC 4283 & E0 & 121750.3 & +293517 & 12.94 & 17.34 & 10.7 & 0.06 & 0 & $1038 \pm 10$ & $119 \pm 05$ & $27 \pm 04$ & 15 \\
\hline NGC 4308 & $\mathrm{E}:$ & 121926.8 & +302110 & 14.16 & 15.88 & $\ldots$ & 0.19 & 0 & $641 \pm 13$ & $62 \pm 08$ & $15 \pm 07$ & 10 \\
\hline NGC 4328 & SA0-: & 122048.0 & +160548 & 14.35 & 16.94 & 16.6 & 0.21 & 90 & $479 \pm 11$ & $36 \pm 05$ & $29 \pm 08$ & 22 \\
\hline$N G C 4344$ & $\mathrm{SpN} / \mathrm{BCD}$ & 122106.1 & +174905 & 13.06 & 18.05 & $\ldots$ & 0.05 & 0 & $1137 \pm 10$ & $24 \pm 07$ & $17 \pm 10$ & 20 \\
\hline$N G C 4352$ & SA0: sp & 122132.2 & +112944 & 13.33 & 17.96 & 16.6 & 0.52 & 102 & $2080 \pm 10$ & $62 \pm 03$ & $68 \pm 08$ & 30 \\
\hline$N G C 4415$ & $\mathrm{~S} 0 / \mathrm{a}$ & 122408.3 & +084247 & 13.66 & 17.63 & 21.4 & 0.11 & 0 & \pm 10 & $41 \pm 03$ & $33 \pm 09$ & 24 \\
\hline NGC 4431 & $\mathrm{SA}(\mathrm{r}) 0$ & 122454.6 & +123403 & 13.66 & 17.63 & 22.9 & 0.39 & 6 & $4 \pm 10$ & $55 \pm 04$ & $60 \pm 09$ & 38 \\
\hline$N G C 4436$ & $\mathrm{dE} 6 / \mathrm{dS} 0, \mathrm{~N}$ & 122509.6 & +123535 & 13.81 & 17.49 & 19.5 & 0.46 & 113 & $1124 \pm 11$ & $40 \pm 04$ & $29 \pm 08$ & 16 \\
\hline$N G C 4474$ & S0 pec: & 122721.7 & +142039 & 12.17 & 19.13 & 23.4 & 0.34 & 80 & $1609 \pm 11$ & $87 \pm 03$ & $88 \pm 04$ & 32 \\
\hline NGC 4476 & $\mathrm{SA}(\mathrm{r}) 0-$ : & 122726.7 & +123727 & 12.89 & 18.40 & 16.2 & 0.28 & 23 & $1970 \pm 11$ & $69 \pm 06$ & $26 \pm 06$ & 18 \\
\hline NGC 4482 & $\mathrm{dE}, \mathrm{N}$ & 122738.4 & +110321 & 13.78 & 17.51 & 33.1 & 0.42 & 128 & $1871 \pm 16$ & $65 \pm 16$ & $\ldots \pm \ldots$ & $\ldots$ \\
\hline$N G C 4578$ & $\mathrm{SA}(\mathrm{r}) 0:$ & 123458.7 & +094947 & 12.14 & 19.15 & 28.2 & 0.26 & 35 & $2273 \pm 12$ & $116 \pm 03$ & $98 \pm 05$ & 35 \\
\hline NGC 4627 & E4 pec & 123933.5 & +325054 & 13.00 & 17.35 & 21.9 & 0.24 & 22 & $542 \pm 16$ & $47 \pm 15$ & $\ldots \pm \ldots$ & $\ldots$ \\
\hline$N G C 5273$ & $\mathrm{SA}(\mathrm{s}) 0$ & 133955.1 & +355417 & 12.35 & 19.23 & 31.6 & 0.21 & 10 & $1064 \pm 11$ & $69 \pm 04$ & $68 \pm 05$ & 23 \\
\hline$N G C 5355$ & E3 & 135139.0 & +403459 & 14.08 & 18.57 & $\ldots$ & 0.36 & 35 & $2344 \pm 11$ & $80 \pm 03$ & $41 \pm 05$ & 20 \\
\hline NGC 5372 & $\mathrm{~S} ?$ & 135305.2 & +585439 & 13.95 & 18.16 & $\ldots$ & 0.43 & 135 & $1717 \pm 13$ & $54 \pm 08$ & $52 \pm 12$ & 12 \\
\hline$N G C 5379$ & S0 & 135356.3 & +595916 & 14.15 & 18.32 & 16.6 & 0.56 & 60 & $1791 \pm 12$ & $53 \pm 08$ & $>115 \pm \ldots$ & 23 \\
\hline$N G C 5473$ & $\mathrm{SAB}(\mathrm{s}) 0-:$ & 140258.9 & +550750 & 12.24 & 20.07 & 20.0 & 0.22 & 160 & $2026 \pm 24$ & $222 \pm 05$ & $161 \pm 08$ & 32 \\
\hline$N G C 5574$ & SB0-? sp & 141824.8 & +032802 & 13.01 & 18.93 & 12.0 & 0.32 & 63 & $1569 \pm 12$ & $77 \pm 03$ & $44 \pm 05$ & 25 \\
\hline NGC 5631 & $\mathrm{SA}(\mathrm{s}) 0$ & 142500.0 & +564825 & 12.25 & 20.33 & 24.5 & 0.00 & 135 & $1929 \pm 11$ & $165 \pm 04$ & $51 \pm 06$ & 32 \\
\hline NGC 5845 & $\mathrm{E}:$ & 150328.8 & +014938 & 13.26 & 18.63 & 3.9 & 0.32 & 139 & $1473 \pm 14$ & $224 \pm 11$ & $151 \pm 11$ & 16 \\
\hline$N G C 5869$ & S0: & 150716.0 & +003934 & 12.44 & 19.48 & 22.9 & 0.30 & 125 & $2074 \pm 16$ & $172 \pm 07$ & $98 \pm 08$ & 32 \\
\hline NGC 6140 & $\mathrm{SB}(\mathrm{s}) \mathrm{cd}$ pec & 162036.0 & +653029 & 12.05 & 18.98 & 49.0 & 0.19 & 50 & $895 \pm 16$ & $36 \pm 13$ & $24 \pm 20$ & 22 \\
\hline
\end{tabular}


Table 1. continued.

\begin{tabular}{|c|c|c|c|c|c|c|c|c|c|c|c|c|}
\hline Object & Type & $\alpha_{1950}$ & $\delta_{1950}$ & $B_{\mathrm{T}}$ & $-M_{B}$ & $r_{\mathrm{e}}$ & $\epsilon$ & $\mathrm{PA}$ & $c z$ & $\sigma_{0}$ & $V_{\max }$ & $r_{\max }$ \\
\hline$(1)$ & $(2)$ & $(3)$ & $(4)$ & $(5)$ & $(6)$ & $(7)$ & $(8)$ & $(9)$ & $(10)$ & $(11)$ & $(12)$ & (13) \\
\hline NGC 6248 & SBd & 164646.7 & +702636 & 14.19 & 17.20 & $\ldots$ & 0.50 & 157 & $1143 \pm 11$ & $\ldots \pm \ldots$ & $\ldots \pm \ldots$ & ... \\
\hline NGC 6339 & $\mathrm{SBd}$ & 171529.7 & +405354 & 13.45 & 19.03 & $\ldots$ & 0.38 & 110 & $2089 \pm 13$ & $66 \pm 11$ & $>20 \pm \ldots$ & 05 \\
\hline NGC 6412 & $\mathrm{SA}(\mathrm{s}) \mathrm{c}$ & 173122.9 & +754426 & 11.95 & 19.60 & 45.7 & 0.10 & 148 & $1313 \pm 26$ & $31 \pm 24$ & $20 \pm 07$ & 22 \\
\hline NGC 6690 & SBcd & 183522.7 & +702855 & 11.94 & $\ldots$ & $\ldots$ & 0.71 & 166 & $477 \pm 12$ & $26 \pm 11$ & $44 \pm 05$ & 33 \\
\hline NGC 7077 & $\mathrm{BCD} / \mathrm{E}$ & 212727.5 & +02 1139 & 14.65 & 16.37 & $\ldots$. & 0.24 & 0 & $1145 \pm 10$ & $33 \pm 04$ & $<15 \pm \ldots$ & 15 \\
\hline NGC 7177 & $\mathrm{SAB}(\mathrm{r}) \mathrm{b} ; \mathrm{HI}$ & 215818.5 & +172949 & 11.69 & 19.75 & 20.4 & 0.34 & 11 & $1131 \pm 12$ & $123 \pm 06$ & $34 \pm 04$ & 25 \\
\hline NGC 7351 & SAB0: & 223842.0 & -044300 & 13.69 & 16.64 & $\ldots$ & 0.43 & 179 & $891 \pm 10$ & $41 \pm 03$ & $66 \pm 08$ & 30 \\
\hline NGC 7457 & $\mathrm{SA}(\mathrm{rs}) 0-?$ & 225836.1 & +295231 & 11.50 & 19.02 & 46.8 & 0.41 & 129 & $833 \pm 10$ & $65 \pm 01$ & $97 \pm 04$ & 60 \\
\hline PGC 28990 & $\mathrm{Sb}-\mathrm{Sc}$ & 095751.2 & +555732 & 16.12 & 15.41 & $\cdots$ & 0.33 & 106 & $1286 \pm 11$ & $22 \pm 10$ & $36 \pm 08$ & 17 \\
\hline PGC 39385 & $\mathrm{E} ?$ & 121436.3 & +30 5514 & 16.00 & 14.48 & $\ldots$ & 0.17 & 0 & $768 \pm 11$ & $19 \pm 06$ & $\ldots \pm \ldots$ & $\ldots$ \\
\hline PGC 41682 & $\mathrm{E} ?$ & 123042.4 & +130802 & 15.12 & 16.98 & $\ldots$. & 0.29 & 23 & $2001 \pm 12$ & $41 \pm 05$ & $15 \pm 05$ & 10 \\
\hline PGC 53521 & S0 & 145618.0 & +021300 & 14.90 & 17.07 & $\ldots$ & 0.37 & 0 & $1810 \pm 12$ & $66 \pm 09$ & $\ldots \pm \ldots$ & $\ldots$ \\
\hline PGC 54452 & S0 & 151300.0 & +022500 & 14.68 & 17.49 & $\ldots$ & 0.19 & 130 & $1907 \pm 11$ & $56 \pm 05$ & $35 \pm 08$ & 15 \\
\hline UGC 01198 & $\mathrm{E} ?$ & 014055.5 & +850039 & 14.90 & 16.50 & $\ldots$ & 0.20 & 70 & $1124 \pm 11$ & $51 \pm 08$ & $\ldots \pm \ldots$ & ... \\
\hline UGC 07436 & dE5 & 121947.4 & +150223 & 14.10 & 17.20 & 21.9 & 0.46 & 125 & $985 \pm 12$ & $47 \pm 08$ & $19 \pm 08$ & 20 \\
\hline UGC 05442 & Im: & 100308.9 & +680418 & 15.54 & 13.52 & 36.2 & 0.50 & 23 & $-18 \pm 14$ & $25 \pm 16$ & $\ldots \pm \ldots$ & $\ldots$ \\
\hline UGC 11539 & $\mathrm{E}$ & 201743.8 & +623200 & 13.76 & 17.98 & $\ldots$ & 0.17 & 3 & $1392 \pm 11$ & $116 \pm 03$ & $48 \pm 08$ & 10 \\
\hline UGC A441 & $\mathrm{BCD} / \mathrm{E}$ & 233509.4 & +295110 & 15.07 & 16.46 & $\ldots$ & 0.14 & 36 & $1382 \pm 10$ & $32 \pm 03$ & $22 \pm 09$ & 10 \\
\hline
\end{tabular}

Notes. Column content (see also the notes on particular objects, below). Column (1): galaxy identification; italic characters correspond to objects observed during the March 1998 run, with the spectrograph setup \#1 (see Sect. 2). Column (2): morphological type from the NED database (status: May 14, 2001), or from BC91 for Virgo dwarfs. Columns (3), (4): coordinates, from the HYPERCAT database (as in Prugniel \& Héraudeau 1998). Column (5): $B_{\mathrm{T}}$, integrated blue magnitude, corrected for Galactic extinction according to Schlegel et al. (1998), and for $k$ term: from HYPERCAT; when unavailable, from the LEDA database (status: May 29, 2001). Column (6): $M_{B}$, absolute $B$ magnitude; the distance moduli are computed using the flowsmoothed velocity from LEDA (Prugniel et al. 1999) corrected for particular streaming as in Prugniel \& Simien (1997), and using $H_{0}=75 \mathrm{~km} \mathrm{~s}^{-1} \mathrm{Mpc}^{-1}$; when unavailable there, from LEDA. Column (7): $r_{\mathrm{e}}$, effective radius in arcsec, corrected for cosmological dimming effect, from HYPERCAT. Column (8): $\epsilon$, ellipticity, derived from parameter $R_{25}$ in LEDA. Column (9): PA, position angle of major axis, in degrees (North through East), as determined on an image of the Digitized Sky Survey, or from LEDA; for Virgo members: from Binggeli \& Cameron (1993). The following columns are results of the present study. Column (10): $c z$, heliocentric radial velocity, in $\mathrm{km} \mathrm{s}^{-1}$. Column (11): $\sigma_{0}$, central velocity dispersion, in $\mathrm{km} \mathrm{s}^{-1}$. Column (12): $V_{\text {max }}$, maximum rotation velocity, in $\mathrm{km} \mathrm{s}^{-1}$ (the $>$ sign indicates that the rotation is still rising at the outermost point, or that the PA of the slit was inclined with respect to the major axis). Column (13): $r_{\max }$, the radius defining the range $\left(-r_{\max }, r_{\max }\right)$ in which $V_{\max }$ was measured, in arcsec.

Notes on particular objects. IC 3328: Cols. (8) and (9) from Jerjen et al. (2000). UGC 05442: Col. (5) from Bremnes et al. (1998), Col. (6) derived from Col. (5) with the same distance modulus as M 81 (28.71: LEDA), Col. (9) from the HYPERCAT FITS archive.

\subsection{Result presentation}

Determinations of the heliocentric radial velocity $c z$, of $\sigma_{0}$, and of $V_{\max }$ (together with the radius $r_{\max }$ of the measurement range) are listed in the last columns of Table 1 . The $V(r)$ and $\sigma(r)$ profiles are presented in Fig. 2, and also in Table 4, which is proposed in electronic form only. For a few galaxies observed with both instrumental setups, only results obtained with setup \#2 are presented: they are more accurate and, especially, the kinematical profiles are deeper; the $\sigma_{0}$ values from setup \#1, however, were found useful for comparison purposes (see Sect. 3.3).

As in Papers III to V, we have followed a convention for the actual position angle PA of the spectrograph slit (as listed in Table 2): for $0<\mathrm{PA}<180^{\circ}, r<0$ corresponds to the eastern side of the galaxy, for $180<\mathrm{PA}<360^{\circ}$, $r<0$ corresponds to the western side, and for $\mathrm{PA}=0^{\circ}$, $r<0$ is to the North.

Tables 1, 2 and 4 are available from the CDS. The data presented here, together with those of the preceding papers of the series, are also available from the HYPERCAT database (Prugniel et al. 1999), at http://www-obs . univ-lyon1.fr/hypercat/.

\subsection{Internal and external comparisons}

It is useful to compare the $\sigma_{0}$ measurements of four dwarf galaxies observed with both setups: IC 3328, IC 3461, IC 3653, and NGC 4436; the dispersions obtained with setup \#1 are, respectively, $35 \pm 8,53 \pm 12,51 \pm 3$, 
Table 3. $\sigma_{0}$ determinations (Papers I to VI) compared to other sources.

\begin{tabular}{lrrrl}
\hline Range & $n$ & Shift & \multicolumn{2}{c}{ Scatter } \\
\hline$(1)$ & $(2)$ & $(3)$ & \multicolumn{2}{c}{$(4)$} \\
\hline all values & 97 & -2 & 17 & $(14 \%)$ \\
$\sigma_{0} \geq 100$ & 81 & -1 & 18 & $(13 \%)$ \\
$\sigma_{0}<100$ & 16 & -5 & 13 & $(27 \%)$ \\
$\sigma_{0}<60$ & 8 & 3 & 11 & $(31 \%)$ \\
\hline
\end{tabular}

Notes. Column (1): range of $\sigma_{0}\left(\right.$ in $\left.\mathrm{km} \mathrm{s}^{-1}\right)$ in the literature set. Column (2): $n$, number of objects in the subsample defined by Col. (1). Column (3): zero-point shift (our values minus literature values). Column (4): estimated rms uncertainty (in $\mathrm{km} \mathrm{s}^{-1}$ ) on an individual measurement, assuming that errors are comparable in both sets; in parentheses, average relative uncertainty on a measurement.

and $46 \pm 15 \mathrm{kms}^{-1}$, fairly close to those of setup \#2. A crude analysis of this very small set of data suggests that there is no systematic shift, and that the average internal relative uncertainty on an individual measurement is $\lesssim 10 \%$.

In our merged sample of $\sigma_{0}$ measurements (Papers I to VI), there are 97 galaxies for which another determination is found in the literature. The results of the comparison are presented in Table 3 . We note the absence of significant systematic discrepancies in all $\sigma_{0}$ ranges. The estimated relative uncertainty increases for faint galaxies: this is not unexpected, since small dispersions are intrinsically more challenging to determine; our measurements must suffer from this, but likely not as much as the mean data from the literature, which are less homogeneous (in both instrumental characteristics and reduction processes).

The reliability of our velocity dispersions over a wide range of surface brightnesses is indicative that our rotation measurements are also free of significant biases. Comparison of our rotation curve for NGC 3377 with that of Bender et al. (1994), up to $r=35^{\prime \prime}$, is satisfactory.

\subsection{Spatial-resolution effects}

The central velocity dispersion and the inner rotation velocity are influenced by the atmospheric smearing and the limited spatial resolution of the instrument. Therefore, the interest of comparing the raw values of $\sigma_{0}$, as above, may be questioned, especially for objects with small angular and intrinsic dimensions, and this point deserves close examination.

To begin with, let us recall that the photometric profile of early-type galaxies is conveniently approximated by the well-known $r^{1 / n}$ luminosity law (Sérsic 1968), with the parameter varying, statistically, from $n \simeq 4$ for bright ellipticals down to $n=1$ or 2 for dwarfs. For this parametric family, Prugniel \& Simien (1997) analyzed the kinematics of a particular sub-class: isotropic, spherical (and therefore non-rotating) systems. Their Fig. 11 shows the luminosity-weighted velocity dispersion integrated within a radius $r$, as a function of $r$; to a first approximation, dependence of the apparent $\sigma_{0}$ on atmospheric conditions and setup characteristics is provided by this graph. For the typical case of a small galaxy with $r_{\mathrm{e}}=10^{\prime \prime}$ and $n=2$, the relative uncertainty on $\sigma_{0}$ is $\sim 5 \%$.

Stellar rotation, if fast enough, can also be a source of error on the measured $\sigma_{0}$. We have simulated its effect on a similar, but flattened, galaxy with a rotation plateau of $V_{\max }=\sigma_{0}$ starting at $0.5 r_{\mathrm{e}}$; to do this, we contaminated the central spectrum providing $\sigma_{0}$ by shifted and luminosity-weighted spectral components: the additional uncertainty on the dispersion is $\lesssim 20 \%$.

We note that an accurate determination of the resolution effects should actually involve, for each individual galaxy, its true photometric profile and a complete modeling of its kinematics. Compared to this, all the above estimates are crude, but they provide reassuring evidence that the kinematical parameters presented are not affected by strong, uncontrolled biases.

\section{Discussion}

\subsection{Statistics}

The results of our reductions are summarized as follows:

- We have measured the central velocity dispersion $\sigma_{0}$ for 72 objects, including 66 early-type galaxies and the bulge of six spirals: in the literature, the $\sigma_{0}$ value was still unavailable for 42 of these objects. For 59 galaxies, we have also determined the $\sigma(r)$ and $V(r)$ profiles along the major axis; we have measured the maximum rotation velocity $V_{\max }$ (or set a limit on it), a parameter which is first presented here for 63 of these objects;

- Thanks to the improved instrumentation used in this work, we have obtained new data on 19 nearby earlytype dwarfs: four in the Local Group, and 15 in the Virgo cluster with a magnitude in the range $-17.6<M_{B}<-15.6$. For all of them, apart from one (NGC 4482), we determined $\sigma_{0}$ and the level of rotation. No previous measurements of $c z$ were available for IC 781, IC 783A, and UGC 5442. We note that these 15 Virgo members lie at the bright end of the dwarf sequence (BC91), and that extending these measurements to the main region of this sequence would be much more challenging: it would therefore be out of our present reach;

- The final sample presented in Papers I through VI now includes 187 galaxies, the wide majority of them being intermediate-luminosity Es and S0s. Although no completeness of any kind is claimed, this already represents a fair fraction of the currently available data on stellar rotation.

\subsection{Comments on particular objects}

Several dwarf galaxies (in the Local Group, in the M 81 group, and in Virgo) deserve a specific comment, as below. 
NGC 205. We find $\sigma_{0}=20 \pm 1 \mathrm{~km} \mathrm{~s}^{-1}$ : this is consistent with the values of $14 \mathrm{~km} \mathrm{~s}^{-1}$ (Carter \& Sadler 1990), $16 \pm 4 \mathrm{~km} \mathrm{~s}^{-1}$ (PC93), and $30 \pm 5 \mathrm{~km} \mathrm{~s}^{-1}$ (BPN91). Our deep major-axis velocity profile extends out to a radius of more than $250^{\prime \prime}$, with a plateau at a significant level of rotation; the minor-axis rotation is $<5 \mathrm{~km} \mathrm{~s}^{-1}$ for $r<120^{\prime \prime}$. This contrasts with the BPN91 major-axis velocity profile, which has about half the radial extent, and which does not show any rotation. We note that our rotation plateau starts at $r \simeq 1.3 r_{\mathrm{e}}$, contrasting with the case of the compact $\mathrm{M} 32$, where $V_{\max }$ is reached at $r \simeq 0.2 r_{\mathrm{e}}$ on our spectra. For NGC 205, therefore, and presumably for other dwarfs with a shallow luminosity profile, determination of the plateau is therefore critically dependent on the radial range of the spectra. This point will be discussed in Sects. 5.3 and 5.4 .

NGC 147 and NGC 185. At variance with NGC 205, poor sky subtraction prevented us from measuring rotation, if any. We have kept these two objects in Table 1 for the interest of the inner region only.

IC 3328. Recently, this object classified $\mathrm{dE} 1, \mathrm{~N}$ has revealed a low-contrast, tightly wound spiral structure (Jerjen et al. 2000), thus suggesting that it is actually a misclassified dSO. Our rotation curve covers a fair fraction of the spiral-pattern area; assuming that our measured $V_{\max }$ comes from a flat, cold component, and adopting the inclination determined by the above authors, the corresponding circular rotation in the equatorial plane is $38 \pm 12 \mathrm{~km} \mathrm{~s}^{-1}$. From Sandage (2000) and references therein, the circular velocities at the faint end of the average Tully-Fisher relation are 49 and $44 \mathrm{~km} \mathrm{~s}^{-1}$, respectively, for small-bulged $\mathrm{Sa}$ and for $\mathrm{Sb}$ spirals with the same $M_{B}$ as IC 3328; compared to these values, our determination is slightly lower but not incompatible, and this provides additional support to the assumption that the geometry is disky.

UGC 5442. Also known as K 64 or KDG 64 , this faint dwarf of the $\mathrm{M} 81$ group has $M_{B} \simeq-13.5$. It features a compact peak of luminosity at a couple of arcsec from the mean isophotal center and, on HST-WFPC2 images, Karachentsev et al. (2000) recognized a background galaxy; on examining the same images, we noticed that it is a flattened galaxy with an apparent diameter barely larger than $1^{\prime \prime}$. This object is present on our major-axis spectra, where it contributes significantly to the flux near the center of UGC 5442; but since its redshift must be considerably higher, it is unlikely to have any effect on the Fourier algorithm, apart from enhancing the noise level. Thus, on averaging the spectra within $\pm 12^{\prime \prime}$ from the center, our measurement of $\sigma_{0}=25 \pm 16 \mathrm{~km} \mathrm{~s}^{-1}$, although inaccurate, should not be biased. We find for UGC 5442 an heliocentric velocity of $-18 \pm 14 \mathrm{~km} \mathrm{~s}^{-1}$, consistent with its belonging to the $\mathrm{M} 81$ group.

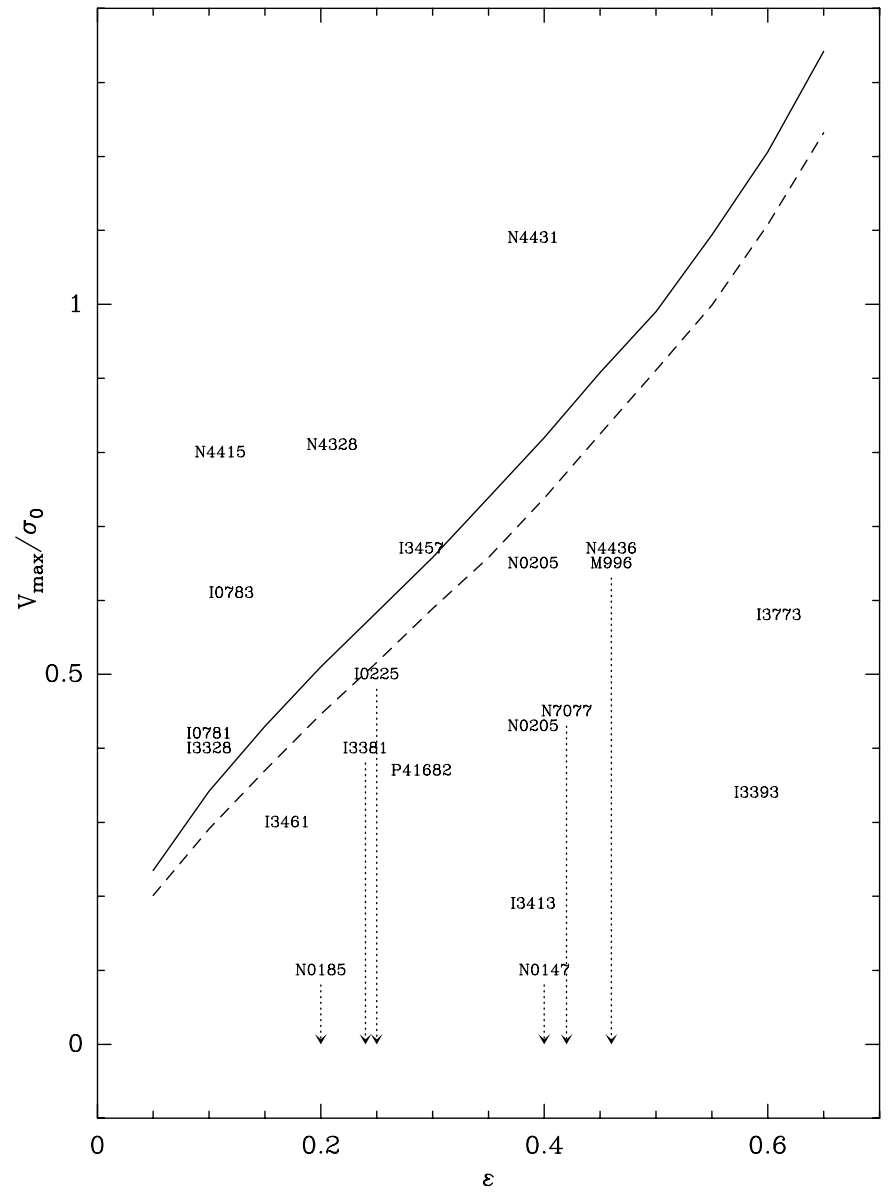

Fig. 1. The $V_{\max } / \sigma_{0}$ kinematic test for a sample of dwarf earlytype galaxies (see Sect. 5.3). Abscissae: apparent ellipticity, from Col. (8) of Table 1; ordinates: ratio of maximum rotational velocity to central dispersion; downward arrows: upper limits. Solid line: isotropic model for an oblate spheroid (Binney 1978); dashed line: same model, for a triaxial spheroid with "representative" axial ratios. The two determinations of NGC 205 refer to two assumed values of $\sigma_{0}$ : either the very central measurement (the bottom of the "dispersion well", or an average at $r \simeq 15^{\prime \prime}$ (which may be more representative of the global dynamical behavior). For the sake of clarity, galaxy identificators have been shortened

\subsection{The kinematical status of dwarf galaxies}

Since the current sample, although incomplete, provides a significant improvement over data available so far, it can be used to check previous suggestions concerning the dynamics and nature of these objects; we present here a preliminary analysis.

Figure 1 locates the dEs on the $V_{\max } / \sigma_{0}$ versus $\epsilon$ diagram (Binney 1978). We have defined a subsample of 20 early-type galaxies with absolute magnitudes in the range $-17.6 \leq M_{B} \leq-14.0$, including 15 Virgo members, and our four Local-Group members. The diagram leads to the following remarks:

- The present sample is far from being dominated by marked anisotropy; types $\mathrm{dE}$ and $\mathrm{dSO}$ are almost 
F. Simien and Ph. Prugniel: Kinematical data on early-type galaxies. VI.
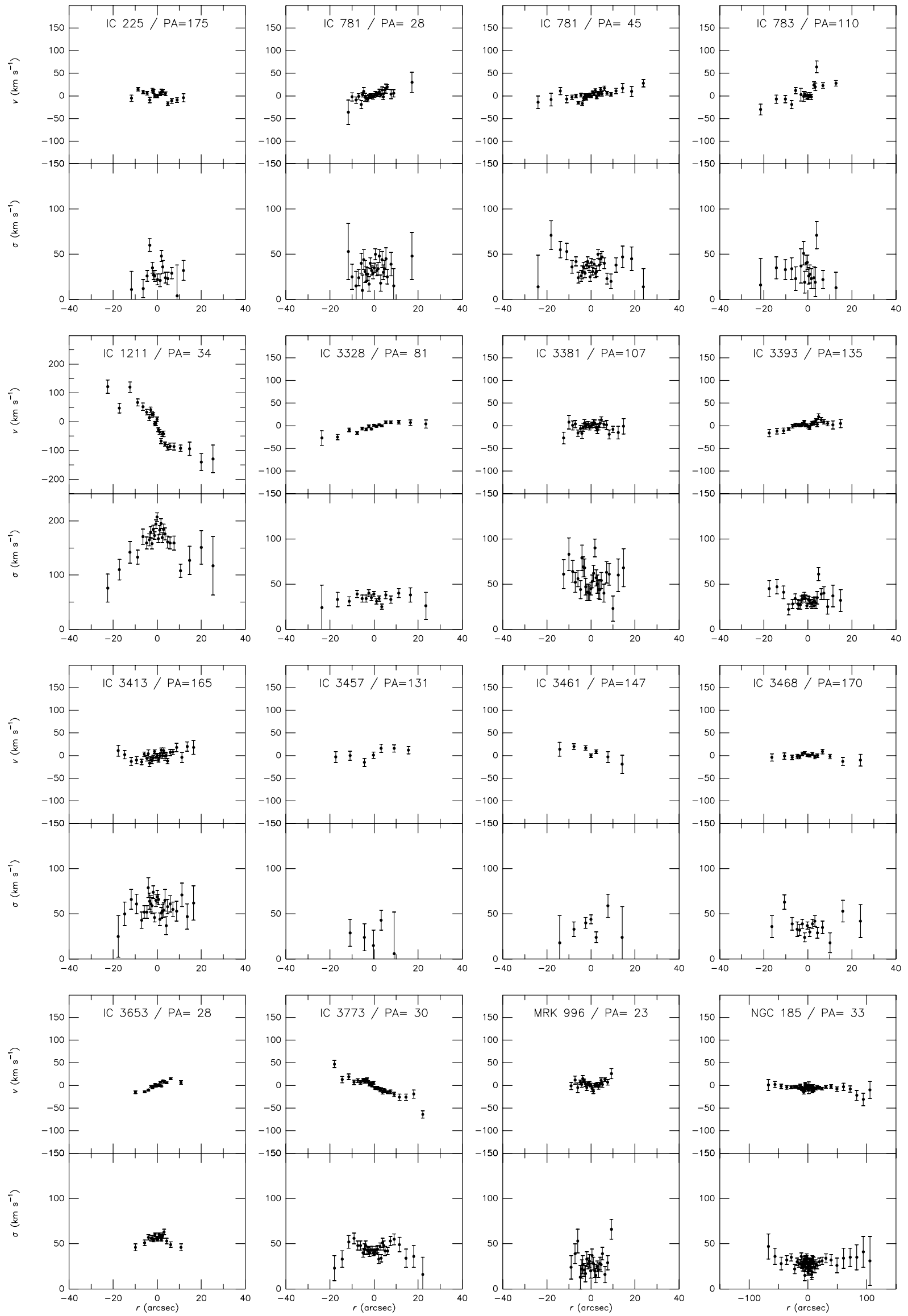

Fig. 2. Profiles of rotation velocities and velocity dispersions. 

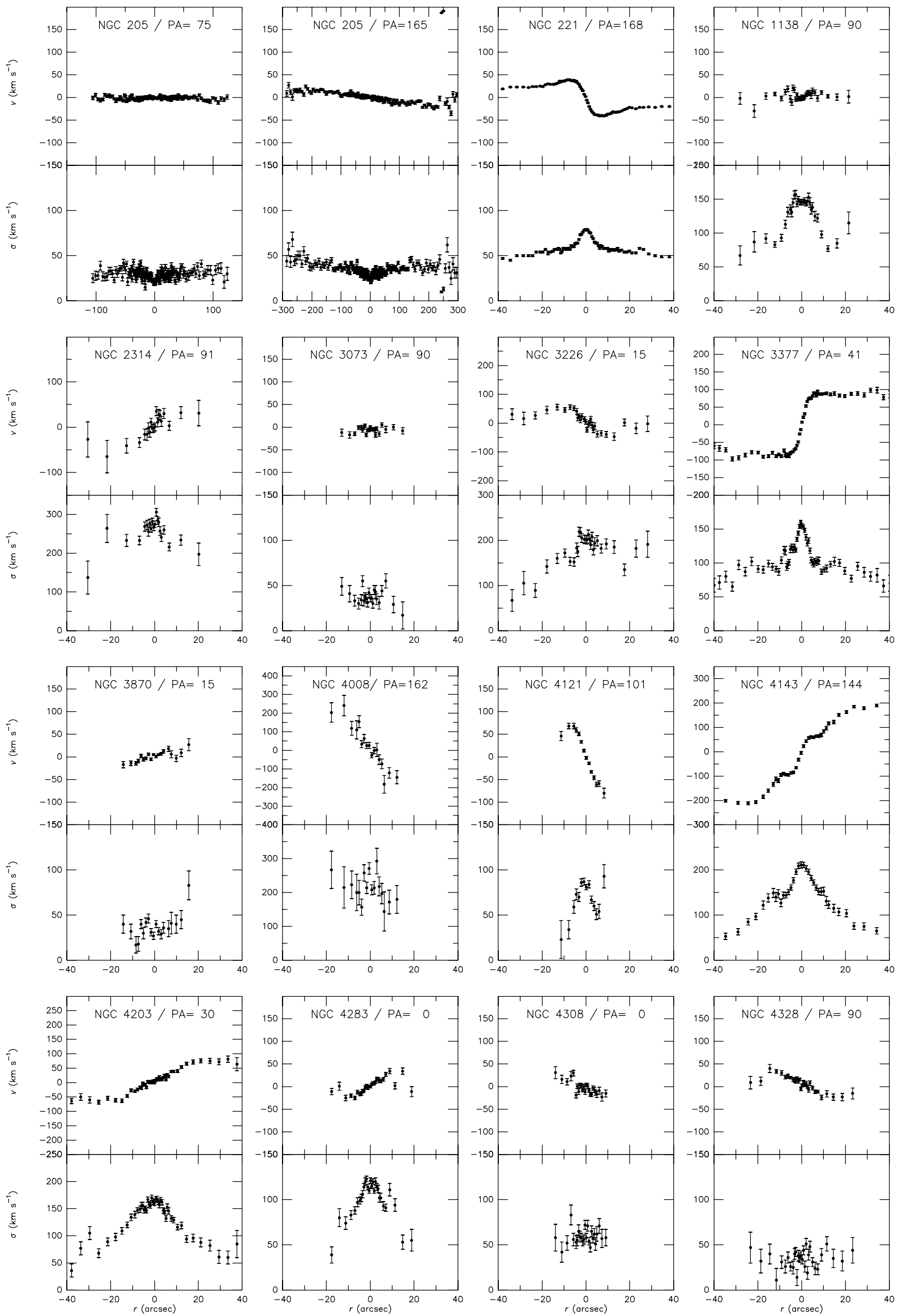

Fig. 2. continued. 

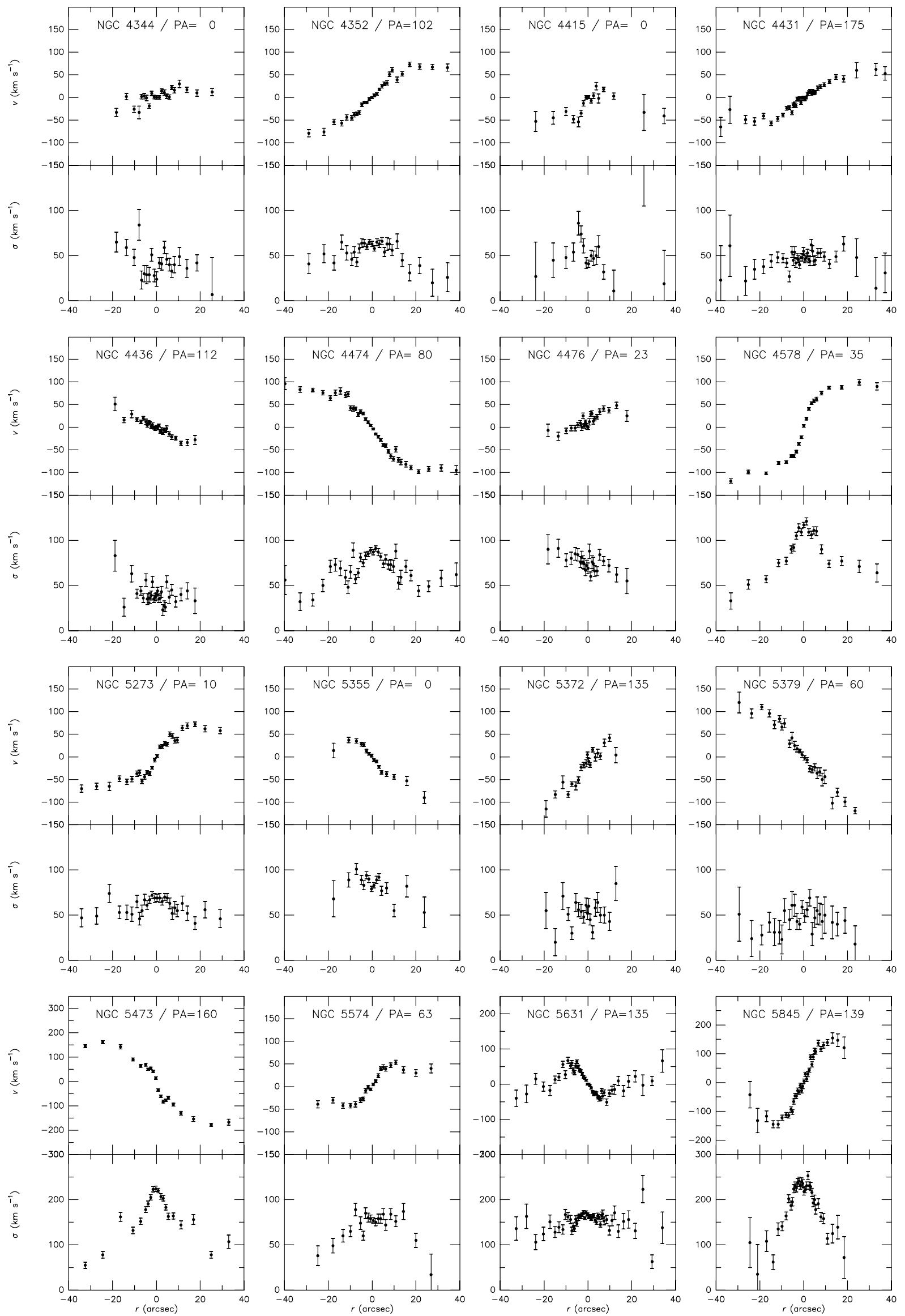

Fig. 2. continued. 

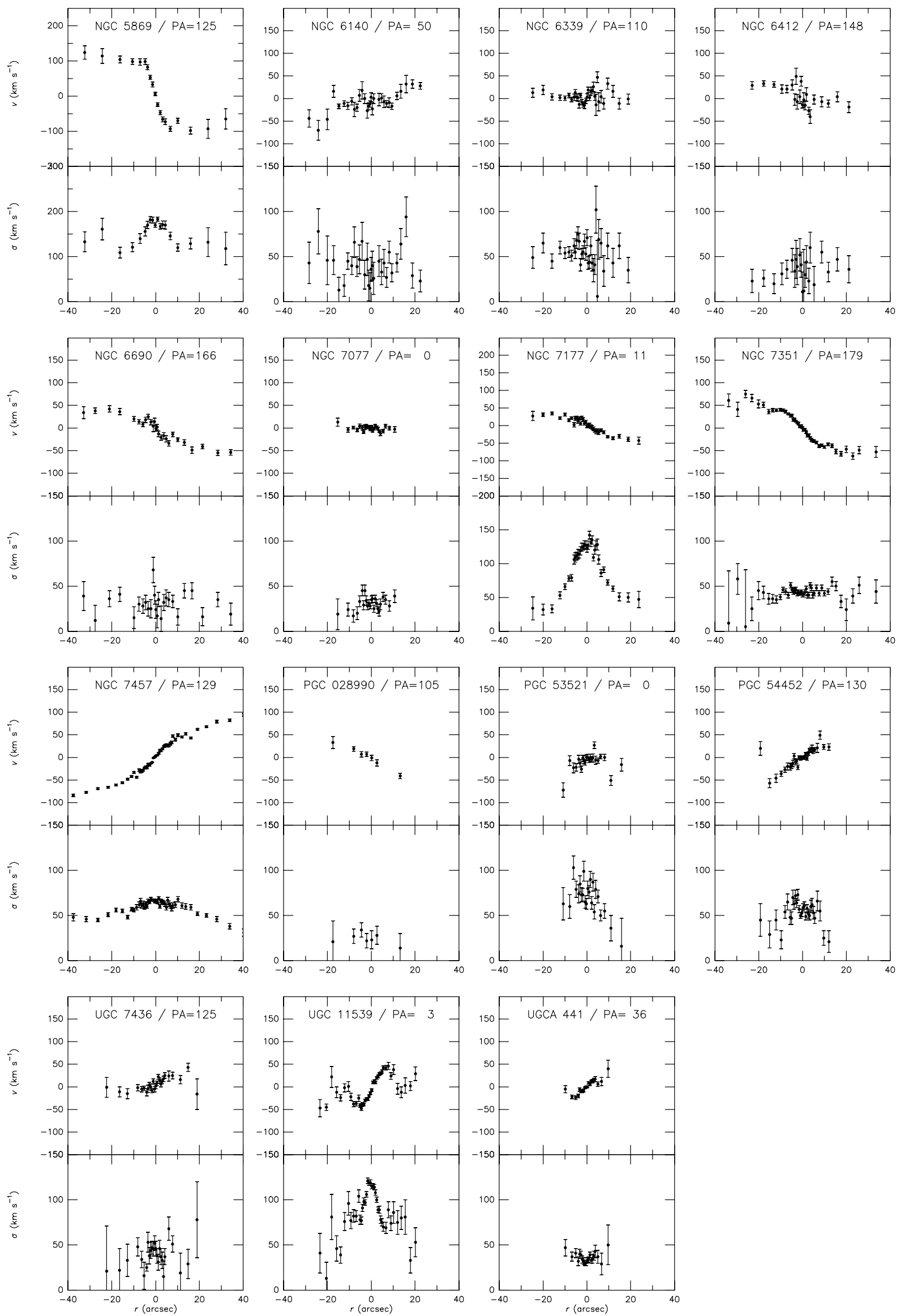

Fig. 2. continued. 
evenly distributed in the "isotropic/anisotropic" dichotomy;

- Bender et al. (1991) and Bender \& Nieto (1990) strongly suggested that none of the galaxies with $M_{B} \gtrsim-17.6$ (for our adopted $H_{0}$ value) and with a low surface brightness, can be considered as close to isotropy. It turns out that many objects in Fig. 1 provide counter-examples. NGC 205 is likely the most revealing one, since an opposite conclusion about its dynamical status has only been a matter of deeper observations;

- BC91 suggest that most, if not all, bright dwarfs (which are nucleated) may actually be disk systems, and they consider spectroscopy as a potential arbiter. Our diagram does not rule out their hypothesis. On the one hand, the position of IC 3328 in Fig. 1, as well as the arguments presented in Sect. 5.2, concur with its newly unveiled morphology; and several additional dEs are fast rotators. But, on the other hand, flattened objects like IC 3773 (dS09, N) and IC 3393 $(\mathrm{dE} 7, \mathrm{~N})$ feature a small measured $V_{\max }$. One can wonder if this is intrinsic or not; again, the extent of the available kinematic profile may be pivotal in settling the question.

\subsection{Concluding remarks}

The initial aim of this series was the systematic publication of all our kinematical observations on spheroidal systems, mostly intermediate-luminosity objects; our recently increased interest in dwarf galaxies, whose low velocity dispersion is more difficult to determine, led us to examine the reliability of our measurements, and to compare them to other sources - this has been done for dwarfs, but also for the entire sample of the six papers. These comparisons did not bring out significant systematic errors.

Among the targeted dwarfs, NGC 205 showed a new characteristic: a significant fraction of rotational energy. Since this well-observed object has so far played the role of an archetypal, anisotropic dwarf not supported by rotation, our result is not devoid of implications. In particular, it added momentum to the test on the kinematical status of dwarf galaxies. But it also provided a strong incentive to investigate this remarkable object more throughly. When complemented with surface-photometry data, our kinematical profiles will allow us to test the relevance of a complete, classical (axisymmetric and isotropic, in a first step) dynamical model, thus relying on much more than the rotation-to-dispersion ratio alone. This will give an opportunity to look at colors and spectral indices, with the aim of disentangling the kinematic and stellar-population effects. These applications are clearly beyond the scope of the present paper, and we hope to be able to present the results in the near future.

It is well-known that, as a kinematical test, the $V_{\max } / \sigma_{0}$ ratio as a function of the apparent flattening is not the most powerful one; nevertheless it is available for galaxies with small angular dimensions and/or scarce data, and it provides a statistically valuable indicator. As stated above, our preliminary analysis of this ratio for dwarfs appears revealing. If the trend toward less anisotropy is confirmed, it would have significant implications for the formation and evolution of a full class of objects. Presently, kinematical data on dEs are still fragmentary, and we expect to continue the observations in order to build up a larger, less biased sample. In this task, the knowledge that rotation should sometimes be measured at radii larger than usually expected may help in avoiding a substantial bias.

Note added in proofs. Our results on the kinematics of dwarfs are in agreement with those recently published by De Rijcke et al. (2001).

Acknowledgements. We are indebted to the telescope operators at the Observatoire de Haute-Provence for their help in collecting the data, and to the PNG for its support. We thank an anonymous referee for his/her many valuable comments on an earlier version of the manuscript. We have made use of the LEDA database (http://leda.univ-lyon1.fr). This research has also made use of the NASA/IPAC Extragalactic Database (NED) which is operated by the Jet Propulsion Laboratory, California Institute of Technology, under contract with the National Aeronautics and Space Administration. We thank the Archive Service of the Space Telescope Science Institute for kindly making HST frames available to us. We have made use of the ESO/ST-ECF first Digitized Sky Survey (DSS). The DSS was produced at the Space Telescope Science Institute under U.S. Government grant NAG W-2166; its images are based on photographic data from the Oschin Schmidt Telescope on Palomar Mountain and the UK Schmidt Telescope. The plates were processed into the present form with the permission of these institutions. The National Geographic Society - Palomar Observatory Sky Atlas was made by the California Institute of Technology with grants from the National Geographic Society. The Oschin Schmidt Telescope is operated by the California Institute of Technology and Palomar Observatory. The UK Schmidt Telescope was operated by the Royal Observatory Edinburgh, with funding from the UK Science and Engineering Research Council (later the UK Particle Physics and Astronomy Research Council), until 1988 June, and thereafter by the Anglo-Australian Observatory; plates of the Sky Atlas Equatorial Extension are from the UK Schmidt.

\section{References}

Bender, R., Burstein, D., \& Faber, S. M. 1992, ApJ, 399, 462 Bender, R., \& Nieto, J. L. 1990, A\&A, 239, 97

Bender, R., Paquet, A., \& Nieto, J. L. 1990, A\&A, 246, 349

Bender, R., Saglia, R. P., \& Gerhard, O. E. 1994, MNRAS, 269, 785

Binggeli, B., \& Cameron, L. M. 1991, A\&A, 252, 27 (BC91)

Binggeli, B., \& Cameron, L. M. 1993, A\&AS, 98, 297

Binney, J. 1978, MNRAS, 183, 501

Bremnes, T., Binggeli, B., \& Prugniel, Ph. 1998, A\&AS, 129, 313

Carter, D., \& Sadler, E. 1990, MNRAS, 245, 12

De Rijcke, S., Dejonghe, H., Zeilinger, W. W., \& Hau, G. K. T. 2001, ApJ, 559, L21 
Jerjen, H., Kalnajs, A., \& Binggeli, B. 2000, A\&A, 358, 845

Karachentsev, I. D., Karachentseva, V. E., Dolphin, A. E., et al. 2000, A\&A, 363, 117

Peterson, R. C., \& Caldwell, N. 1993, AJ, 105, 1411

Prugniel, Ph., Golev, V., \& Maubon, G. 1999, A\&A, 346, L25

Prugniel, Ph., \& Héraudeau, Ph. 1998, A\&AS, 128, 299

Prugniel, Ph., Maubon, G., \& Simien, F. 2001, A\&A, 366, 68

Prugniel, Ph., \& Simien, F. 1994, A\&A, 282, L1

Prugniel, Ph., \& Simien, F. 1996, A\&A, 309, 749

Prugniel, Ph., \& Simien, F. 1997, A\&A, 321, 111
Sandage, A. 2000, PASP, 112, 504

Schlegel, D. J., Finkbeiner, D. P., \& Davis, M. 1998, ApJ, 500, 525

Sérsic, J.-L. 1968, Atlas de Galaxias Australes, Observatorio Astronómico de Córdoba

Simien, F., \& Prugniel, Ph. 1997a, A\&AS, 122, 521 (Paper I)

Simien, F., \& Prugniel, Ph. 1997b, A\&AS, 126, 15 (Paper II)

Simien, F., \& Prugniel, Ph. 1997c, A\&AS, 126, 519 (Paper III)

Simien, F., \& Prugniel, Ph. 1998, A\&AS, 131, 287 (Paper IV)

Simien, F., \& Prugniel, Ph. 2000, A\&AS, 145, 263 (Paper V) 